\title{
SOME LOCAL-GLOBAL RESULTS IN FINITE TRANSFORMATION GROUPS
}

\author{
AMIR H. ASSADI
}

\begin{abstract}
The main theorem is a converse to the localization theorem in equivariant cohomology, using the Sullivan fixed point conjecture. In applications, we prove that certain fibrations over $B G$ are fiber homotopy equivalent to the Borel constructions of finite dimensional (finitely dominated) $G$-spaces.
\end{abstract}

Introduction. Let $G$ be a finite group, and let $X$ be a $G$-space. The Borel equivariant cohomology of $X$ is denoted by

$$
H_{G}^{*}(X) \equiv H^{*}\left(E_{G} \times_{G} X ; \mathbf{F}_{p}\right),
$$

where $E_{G} \times_{G} X \stackrel{\pi}{\rightarrow} B G$ is the twisted product associated to the universal bundle $E_{G} \rightarrow B G$. For a $p$-elementary abelian group, i.e.

$$
G \simeq(\mathbf{Z} / p \mathbf{Z})^{n}, \bigoplus_{k \geq 0} H^{2 k}\left(B G ; \mathbf{F}_{p}\right)_{\mathrm{red}} \simeq \mathbf{F}_{p}\left[t_{1}, \ldots, t_{n}\right]
$$

is a polynomial algebra denoted by $H_{G}$. Then $H_{G}^{*}(X)$ is a graded $H_{G}$-module and the localization $S^{-1} H_{G}^{*}(X)$ is obtained by inverting nonzero elements of $H^{2}\left(B G ; \mathbf{F}_{p}\right)$. According to the Borel localization theorem [B], for a finite dimensional $G$-space $X$, the inclusion of the fixed points $j: X^{G} \rightarrow X$ induces an $S^{-1} H_{G^{-}}$isomorphism $j^{*}: S^{-1} H_{G}^{*}(X) \cong S^{-1} H_{G}^{*}\left(X^{G}\right)$. Further refinements of localization in equivariant cohomology with deep applications are due to Quillen

[Q] and W. Y. Hsiang $[\mathbf{H s}]$, where the $p$-elementary abelian groups (and tori) play a key role in an algebro-geometric setting. In $[\mathbf{B}, \mathbf{H s}, \mathbf{Q}]$ and related developments, the hypothesis $\operatorname{dim} X<\infty$ is indispensable since the localization theorem fails if $\operatorname{dim} X=\infty$. In [Su] D. Sullivan pointed to new directions. Let $\operatorname{Map}\left(E_{G}, X\right)$ be the mapping space together with the conjugation $G$-action $f^{g}(x)=g^{-1} f(g x)$, so that the subspace of equivariant maps $\operatorname{Map}_{G}\left(E_{G}, X\right)$ and the fixed point set $\operatorname{Map}\left(E_{G}, X\right)^{G}$ coincide. In 1970 Sullivan conjectured that for $G=\mathbf{Z} / 2 \mathbf{Z}$, the inclusion $X^{G} \rightarrow \operatorname{Map}_{G}\left(E_{G}, X\right)$ (via constant maps) induces a weak homotopy equivalence after 2 -adic completion [Su]. In 1982, H. Miller's proof of the important special case $X=X^{G}$ marked a new era in transformation groups and homotopy theory $[\mathbf{M}]$. As pointed out by $\mathrm{H}$. Miller,

Received by the editors August 13, 1987, and, in revised form, October 14, 1987.

1980 Mathematics Subject Classification (1985 Revision). Primary 57S17, 55M35; Secondary 55P10, $55 \mathrm{U} 15$.

Key words and phrases. Equivariant cohomology, $G$-spaces, completion, localization.

The author is grateful to NSF, Max-Planck-Institute für Mathematik (Bonn), and the University of Wisconsin Graduate School for partial financial support. 
the general form of the conjecture as stated in [Su] needs technical modification to be true. Recently, G. Carlsson [C], J. Lannes [L], and H. Miller [Mm] have announced independent proofs of modified versions of the Sullivan conjecture for $G=p$-group. This leads to a converse to the localization theorem and its corollaries below.

Notation and conventions. All spaces are paracompact. $p=$ prime integer, $\mathscr{P}_{p}=$ the set of $p$-subgroups of $G$;

$$
\begin{gathered}
\mathscr{C}_{p}=\left\{X: \operatorname{dim} X<\infty \text { and } \operatorname{dim} H_{*}\left(X ; \mathbf{F}_{p}\right)<\infty\right\} ; \\
\mathscr{C}_{0}=\left\{X: X \text { is weakly equivalent to some } Y \in \bigcap_{P \||G|} \mathscr{C}_{p}\right\} ; \\
\mathscr{C}_{G}=\left\{G \text {-space } X: \forall H \in \mathscr{P}_{p} \forall p \| G \mid, X^{H} \in \mathscr{C}_{p} \text { and } X \in \mathscr{C}_{p}\right\} ;
\end{gathered}
$$

$\mathscr{R}_{p}=a$ suitable $p$-completion functor such as $[\mathbf{B K}, \mathbf{M m}]$ and $\rho^{X}: X \rightarrow$ $\mathscr{R}_{p}(X)$ the natural map; $\operatorname{Top}\left(\mathscr{R}_{p}\right)=\left\{X: H_{*}\left(\rho^{X}: \mathbf{F}_{p}\right)\right.$ is an isomorphism $\}=$ " $p$-good spaces" of [BK] if $\mathscr{R}_{p}$ is the Bousfield-Kan completion. $E$ is a free contractible $G$-space, i.e. $E=E_{G}$.

1. A converse to the localization theorem. In problems involving the construction of $G$-actions (e.g. on manifolds or finite CW complexes), one often proceeds by homotopically constructing a fibration $Y \rightarrow B G$ with fiber homotopy equivalent to the given space $X$, so that the $G$-covering $\tilde{Y}$ has a free $G$-action and $\tilde{Y} \simeq X$. In practice, $\operatorname{dim} \tilde{Y}=\infty$ and one needs to know:

When does there exist a finite dimensional (finitely dominated) $G$-space $K$ such that $\tilde{Y} \simeq_{G} E_{G} \times K$ (i.e. $G$-homotopy equivalent).

To construct a "finite dimensional model $K$ " from the fibration $Y \rightarrow$ $B G$, one encounters questions intimately related to the Sullivan conjecture. The valid statements of the Sullivan conjecture involve completion functors of Bousefield and Kan [BK] in [C, L ] and the version of Dwyer-MillerNeisendorfer in $[\mathbf{M m}]$. For $X \in \mathscr{C}_{G}$, let $F=X^{G}$. Let $\bar{\eta}: F \rightarrow \operatorname{Map}_{G}(E, X)$ and $\hat{\eta}=$ adjoint of the composition

$$
E \times \mathscr{R}_{p}(F) \rightarrow \mathscr{R}_{p}(E) \times \mathscr{R}_{p}(F) \cong \mathscr{R}_{p}(E \times F) \stackrel{\mathscr{R}_{p}(\bar{\eta})}{\longrightarrow} \mathscr{R}_{p}(X) .
$$

We need the following modified version of the Sullivan conjecture.

Theorem (G. Carlson, J. LAnnes, H. Miller). Let $G=\mathbf{Z}_{p}^{n}$ and $X \in \mathscr{C}_{G}$ be connected. Then $\hat{\eta}: \mathscr{R}_{p}(F) \rightarrow \operatorname{Map}_{G}\left(E, \mathscr{R}_{p}(X)\right)$ is a weak equivalence.

The following provides a converse to the localization theorem as well as a characterization of finite dimensional $G$-spaces in terms of localized equivariant cohomology. There is also an analogous theorem for finitely dominated $G$-spaces.

THEOREM 1. Let $G$ be any finite group acting on a simply-connected $G$ space $X \in \mathscr{C}_{0}$. There exists a finite dimensional $G$-space $Y \in \mathscr{C}_{G}$ such that $E \times Y \simeq_{G} E \times X$ and $Y^{H} \in \operatorname{Top}\left(\mathscr{R}_{p}\right)\left(\forall p \forall H \in \mathscr{P}_{p}\right)$ if and only if: 
(A1) For each $P \in \mathscr{P}_{p}$, there exists $F \in \operatorname{Top}\left(\mathscr{R}_{p}\right) \cap \mathscr{C}_{p}$ and $\eta: F \rightarrow$ $\operatorname{Map}_{p}(E, X)$ inducing an isomorphism

$$
\hat{\eta}_{*}: H_{*}\left(F ; \mathbf{F}_{p}\right) \rightarrow H_{*}\left(\operatorname{Map}_{p}(E, X) ; \mathbf{F}_{p}\right) .
$$

(A2) For each pair $Q \subseteq P \in \mathscr{P}_{p}$ with $Q / P \equiv A \cong \mathbf{Z}_{p}^{n}$ and inclusion $j: \operatorname{Map}_{p}\left(E, \mathscr{R}_{p}(X)\right) \rightarrow \operatorname{Map}_{Q}\left(E, \mathscr{R}_{p}(X)\right), S^{-1} H_{A}^{*}(j)$ is an isomorphism.

IDEA OF PROOF. First, proceed for the case $G=\mathbf{Z} / p \mathbf{Z}$ by translating the Sullivan conjecture in terms of the localization theorem of Borel-QuillenHsiang for mapping spaces to obtain the necessary conditions. For the sufficiency, the space $K$ is constructed directly starting with the fixed point set information contained in the space of sections of $E_{G} \times{ }_{G} X \rightarrow B G$. The technical issues involve dealing with completions and localizations. For the general case, the singular set of the candidate $K$ is constructed inductively, using algebraic computations and frequent applications of the projectivity criterion of $[\mathbf{A}, \mathbf{A a}]$ (in its modular form). Using the infinite dimensional $G$-space $\operatorname{Map}\left(E_{G}, X\right)$ as the target, we add free $G$-cells to the singular set to obtain finite dimensional approximations. The final stage reduces to proving the $\mathbf{Z} G$ projectivity of a certain module. According to $[\mathbf{A}, \mathbf{A a}]$, this can be detected by restriction to subgroups isomorphic to $\mathbf{Z} / p \mathbf{Z}$, and $(\mathbf{Z} / p \mathbf{Z})$-projectivity follows from the first case.

2. COROLlARY. Let $X$ be a $G$-space. Then the following are equivalent:

(1) There exists a finite dimensional (finitely dominated) $G$-space $K \in \mathscr{C}_{G}$ such that $E \times X \simeq_{G} E \times K$.

(2) For each representative $p$-Sylow subgroup $P \subset G$, there exists a finite dimensional (finitely dominated) $P$-space $K(P) \in \mathscr{C}_{p}$ such that $E \times X$ is $P$-homotopy equivalent to $E \times K(P)$.

In some cases, condition (2) of the above can be further simplified by using only subgroups isomorphic to $\mathbf{Z} / p \mathbf{Z}$. Theorem 1 is useful in particular in conjunction with Lannes' $T$-functor $[\mathbf{L}]$ in circumstances where the fundamental groups of the related mapping spaces do not cause difficulties, e.g.:

3. COROLLARY. Let $X$ be a simply-connected $G$-space such that $H_{i}(X)=$ 0 for $i \neq 0, n$. Suppose for each $p$-Sylow subgroup $H \subseteq G,\left.H_{n}(X)\right|_{\mathbf{z} H} \cong P \oplus Q$, where $P$ is $\mathbf{Z} H$-projective, and $Q$ is $\mathbf{Z} H$-indecomposable if $|H|=p$ and $Q=0$ otherwise. Then there exists a $G$-space $K, \operatorname{dim} K<\infty$, such that $E_{G} \times X \simeq_{G}$ $E_{G} \times K$.

4. COROLlARY. Let $G$ be any finite group of order $q$. Suppose $Y \stackrel{\eta}{\rightarrow} B G$ is a fibration with fiber $F \in \mathscr{C}_{0}$, such that $H_{*}(F ; \mathbf{Z} / q) \cong H_{*}\left(S^{n} ; \mathbf{Z} / q\right)$. Then $\eta$ is fiber homotopy equivalent to the Borel construction $E_{G} \times_{G} K \rightarrow B G$ where $\operatorname{dim} K<\infty\left(\pi_{1}(F)=0\right)$.

The above corollaries use Theorem 1 to reduce the problem to $p$-elementary abelian groups. Then the results of Lannes $[\mathbf{L}]$ and other computations (e.g. of [CLM]) in conjunction with Lannes' $T$-functor allow one to verify the localization condition on the equivariant mapping spaces. 


\section{REFERENCES}

[A] A. H. Assadi, Varieties in finite transformation groups, Bull. Amer. Math. Soc. (N.S.) 19 (1988), 459-463.

[Aa] , Homotopy actions and cohomology of finite groups, Proc. Trans. Groups, Poznan, 1985, Lecture Notes in Math., vol. 1012, Springer-Verlag, Berlin and New York, 1986, pp. 26-57.

[B] A. Borel et al., Seminar in transformation groups, Ann. of Math. Studies, Princeton Univ. Press, Princeton, N. J., 1962.

[BK] Bousfield-Kan, Homotopy limits, localization, and completion, Lecture Notes in Math., vol. 304, Springer-Verlag, Berlin and New York, 1972.

[C] G. Carlsson, Equivariant stable homotopy and Sullivan's conjecture, preprint, 1986.

[CLM] Cohen-Lada-May, The homology of iterated loop spaces, Lecture Notes in Math., vol. 533, Springer-Verlag, Berlin and New York, 1976.

[Hs] W. Y. Hsiang, Cohomology theory of topological transformation groups, Ergeb. Math. Grenzgeb. Band 85, Springer-Verlag, Berlin and New York, 1975.

[L] J. Lannes, Sur la cohomologie modulo $p$ des p-groupes Abeliens elementaires, preprint, 1986.

[M] H. R. Miller, The Sullivan conjecture on maps from classifying spaces, Ann. of Math. (2) 120 (1984), 39-87.

[Mm] _ , The Sullivan conjecture and homotopical representation theory, Proc. ICM (1986).

[Q] D. Quillen, The spectrum of an equivariant cohomology ring. I, II, Ann. of Math. (2) 94 (1971), 549-572; 573-602.

[Su] D. Sullivan, Geometric topology, Part I (MIT mimeographed notes 1970).

Department of Mathematics, University of Wisconsin, Madison, WISCONSIN 53706

Current address: FORSCHUNGSINSTITUT DER MATHEMATIK, ETH-ZENTRUM, ZÜRICH, SWITZERLAND 